\title{
Remineralization and repair of enamel surface by biomimetic Zn-carbonate hydroxyapatite containing toothpaste: a comparative in vivo study
}

\section{Marco Lelli ${ }^{1+}$, Angelo Putignano ${ }^{2+}$, Marco Marchetti ${ }^{1}$, Ismaela Foltran ${ }^{1}$, Francesco Mangani ${ }^{3}$, Maurizio Procaccini ${ }^{2}$, Norberto Roveri ${ }^{1}$ and Giovanna Orsini ${ }^{2 *}$}

\author{
1 Department of Chemistry "G. Ciamician," Alma Mater Studiorum, University of Bologna, Bologna, Italy \\ ${ }^{2}$ Department of Clinical Sciences and Stomatology, Polytechnic University of Marche, Ancona, Italy \\ ${ }^{3}$ Department of Translational Medicine, Tor Vergata University of Rome, Rome, Italy
}

\section{Edited by:}

Bernhard Ganss, University of

Toronto, Canada

Reviewed by:

Thimios Mitsiadis, University of

Zurich, Switzerland

Simone Grandini, University of

Siena, Italy

*Correspondence:

Giovanna Orsini, Polytechnic

University of Marche, Via Tronto 10,

60126 Ancona, Italy

e-mail:g.orsini@univpm.it;

giovorsini@yahoo.com

tThese authors have contributed equally to this work.
Consumption of acidic foods and drinks and other factors that cause enamel wear are responsible for the daily enamel loss and degradation. Use of some toothpastes that have been showed to possess different properties of remineralisation and/or repair of the enamel surface may help to protect tooth enamel. The aim of this study was to evaluate whether the use of toothpaste containing Zn-carbonate hydroxyapatite (CHA) nanostructured microcrystals may exert remineralization/repair effects of the enamel surface. Two groups of patients, aged between 18 and 75 years, used a $\mathrm{Zn}-\mathrm{CHA}$ nanocrystals-based toothpaste (experimental group) and a potassium nitrate/sodium fluoride toothpaste (active control group) for 8 weeks. At the end of this period, extractions were performed in five subjects per study group. Negative controls consisted of two subjects treated with non-specified fluoride toothpaste. Teeth were processed for morphological and chemical-physic superficial characterizations by means of Scanning Electronic Microscopy with Elementary analysis, X-Ray Diffraction analysis and Infrared analysis. In this study, the use of a $\mathrm{Zn}-\mathrm{CHA}$ nanocrystals toothpaste led to a remineralization/repair of the enamel surface, by deposition of a hydroxyapatite-rich coating. On the other hand, the use of both a nitrate potassium/sodium fluoride and non-specified fluoride toothpastes did not appreciably change the enamel surface. In conclusion, this study demonstrates that the toothpaste containing Zn-CHA nanostructured microcrystals, differently from nitrate potassium/sodium fluoride and non-specified fluoride toothpastes, may promote enamel superficial repair by means of the formation of a protective biomimetic CHA coating.

Keywords: enamel, toothpaste, Zn-carbonate hydroxyapatite nanocrystals, fluoride, repair, remineralization

\section{INTRODUCTION}

Enamel loss as a part of tooth wear has become a common problem in everyday dental practice, which can be caused by erosion (Hooper et al., 2003), attrition and abrasion, being often associated with dentine hypersensitivity (DH) in different teeth (Absi et al., 1987; Addy, 1990; Addy et al., 2007; Addy and West, 2013). The predicted percentage of adults presenting severe tooth wear has increases with age from $3 \%$ at the age of 20 years to $17 \%$ at the age of 70 years (Van't Spijker et al., 2009).

Erosion is currently believed to be the major factor involved in tooth wear, and has been defined as the dissolution of teeth natural hydroxyapatite by either extrinsic or intrinsic acids, which are not originated from bacteria (Scheutzel, 1996; Zero and Lussi, 2000). Attrition describes the wear of teeth at sites of direct contact between teeth, which can be associated with occlusal function and may be exaggerated by parafunctional habits such as bruxism (Smith and Knight, 1984). Abrasion describes the wear of teeth caused by objects, including toothbrush, abrasive toothpastes as well as a variety of habits like pipe smoking (Dababneh et al.,
1999). The combined action of all the above mentioned mechanisms may occur frequently, resulting in the dentine exposure, which might lead to the development of DH by means of the most widely accepted hydrodynamic theory proposed by Brannström (Brannström, 1963; Hefferren, 1976; Groeneveld et al., 1990; Drisko, 2007).

Among the numerous treatment regimens that have been recommended over these years, particular attention has been focused on toothpastes containing potassium salts (Markowitz et al., 1991; Nagata et al., 1994; Peacock and Orchardson, 1995; Orchardson and Gillam, 2000; Poulsen et al., 2006; Bellamy et al., 2009) and, more recently, on a new toothpaste formulation containing zinc $(\mathrm{Zn})$-substituted carbonate-hydroxyapatite (CHA) nanostructured microcrystals (from now on called Zn-CHA toothpaste), which has been shown to produce in vitro remineralization of the altered enamel surfaces and to be effective in closing dentinal tubules (Kuroiwa et al., 1994; Rimondini et al., 2007; Lee et al., 2008; Roveri et al., 2008, 2009a). Indeed, a clinical randomized trial has shown the efficacy of CHA-based toothpastes 
in reducing $\mathrm{DH}$, after 4 and 8 weeks. In particular, this study demonstrated that $\mathrm{Zn}$-CHA toothpaste compared with potassium nitrate/fluoride toothpaste $\left(\mathrm{KNO}_{3} / \mathrm{NaF}\right.$, active control) showed a significant improvement in airblast test scores (mean percentage of reduction 46.0 vs. $29.4 \%$ in controls) and subjective test scores ( 47.5 vs. $28.1 \%$, respectively), with both differences being significant already after 4 weeks (Orsini et al., 2010).

However, this latter trial presented two major limitations consisting in: (a) the true efficacy of both toothpastes could not be assessed, since no morphological analyses were carried out on the dental surfaces; (b) no negative control such as a fluoride toothpaste (Holland et al., 1997) was used. Therefore, the aim of the present study was to investigate (1) whether the in vitro action of $\mathrm{Zn}$-CHA toothpaste, based on the gradual remineralizing/repairing action of the dental surface by means of the deposition of a biomimetic CHA coating (Rimondini et al., 2007; Roveri et al., 2009a) could be confirmed also in vivo, and (2) whether the $\mathrm{Zn}$-CHA toothpaste and the $\mathrm{KNO}_{3} / \mathrm{NaF}$ toothpaste may lead to stable morphological changes of the dental surface. These aims will be assessed by comparing the enamel surface of teeth treated in vivo for 8 weeks with: (I) Zn-CHA toothpaste (experimental group); (II) $\mathrm{KNO}_{3} / \mathrm{NaF}$ toothpaste (active control group); (III) fluoride toothpaste (negative control group).

\section{MATERIALS AND METHODS STUDY DESIGN AND POPULATION}

This study was carried out as the conclusive part of a previously published randomized trial conducted at the Department of Clinical Sciences and Stomatology of the Polytechnic University of Marche, Ancona, Italy (registered in the Australian New Zealand Clinical Trials Registry with the number: 00362190). According to Silverman et al. (1996) inclusion criteria were: hypersensitive area on facial surfaces of the teeth (incisors, cuspids, bicuspids and first molars with exposed cervical dentine), with at least two teeth scoring one or more at the air blast sensitivity test; good periodontal health (no probing depth $>4 \mathrm{~mm}$ ) with no other conditions which might explain their apparent $\mathrm{DH}$; good physical health; age between 18 and 75 years; provision of written informed consent. Exclusion criteria were: chipped teeth, defective restorations, fractured undisplaced cuspids, deep dental caries or large restorations showing pulpal response, deep periodontal pockets, orthodontic appliances, dentures, or bridgework that would interfere with the evaluation of hypersensitivity; periodontal surgery within the previous 6 months; ongoing treatment with antibiotics and/or anti-inflammatory drugs; ongoing treatment for tooth hypersensitivity; pregnancy or lactation (Singal et al., 2005); acute myocardial infarction within the past 6 months, use of a pace-maker, uncontrolled metabolic diseases, major psychiatric disorder, heavy smoking and alcohol or drug abuse.

Eligible subjects were randomized to receive either the new toothpaste formulation (experimental group), containing biomimetic nanocrystals of Zn-substituted CHA, assembled in microparticles (Zn-CHA toothpaste, BioRepair ${ }^{\circledR}$ Plus, Coswell S.p.A., Funo, Bologna, Italy) or a commercially available desensitizing toothpaste (active control group), containing
$5 \% \mathrm{KNO}_{3} / \mathrm{NaF}$ with $1450 \mathrm{ppm}$ fluoride $\left(\mathrm{KNO}_{3} / \mathrm{NaF}\right.$ toothpaste, Sensodyne ProNamel ${ }^{\mathrm{TM}}$, GlaxoSmithKline Consumer Healthcare, Brentford, U.K.). The final protocol was approved by the Ethical Committee of the Polytechnic University of Marche, Ancona, Italy.

The clinical examination of the subjects was performed at baseline, after 4 weeks and after 8 weeks (end of the follow-up). During the visits, a minimum of two and up to four hypersensitive teeth was assessed using the most common and validated stimuli tests: tactile test, airblast test, cold water test and subjective test (Tarbet et al., 1979; Holland et al., 1997; Singal et al., 2005; Orsini et al., 2010).

Among the patients that completed the study, five subjects in the experimental group and five subjects in the active control group were selected for the present study because they needed extractions of sound teeth for orthodontic or prosthetic reasons. The extractions were performed after the end of the follow-up ( 8 weeks). At this time, two further subjects that needed extractions and brushed their teeth using a non-specified fluoride toothpaste were also included (fluoride toothpaste, negative control group).

\section{ANALYSIS OF Zn-SUBSTITUTED CARBONATE-HYDROXYAPATITE (CHA) NANOCRYSTALS AND CHA MICROCLUSTERS CONTAINED IN THE EXPERIMENTAL TOOTHPASTE}

Plate-acicular shaped CHA nanocrystals, about $70-100 \mathrm{~nm}$ in size, were synthesized according to a modification of the method previously reported and patented (Gazzaniga et al., 2006). CHA nanocrystals have been allowed, after synthesis, to grow in the reaction mixture under stirring up to the formation of nanostructured clusters having dimensions ranging from about 0.5 to $3.0 \mu \mathrm{m}$, according to the patented methodology. Then, the stirring was suspended allowing the deposition of CHA nanostructured microclusters isolated by filtration of the solution, repeatedly washed with water, and freeze-dried (Roveri et al., 2009b). CHA nanocrystals as well as the formed microclusters were analyzed by means of SEM, TEM, XRD, FT-IR. Specimens of human enamel served as controls.

\section{MORPHOLOGICAL CHARACTERIZATION}

Scanning Electron Microscopy (SEM) observations were carried out by means of a SEM, Carl-Zeiss EVO, 40 XVP (Oberkoche, Germany) equipped with energy dispersive detector (EDAX) Inca 250 (Oxford, UK), using secondary electrons at $25 \mathrm{Kv}$ and different magnifications. Specimens were mounted on aluminum stubs with a carbon tape and covered by a $10 \mathrm{~nm}$ thick carbon coating, using a coating unit.

Transmission Electron Microscopy (TEM) investigations were carried out by means of a Philips CM 100 (Eindhoven, The Netherland) instrument. The powdered samples were ultrasonically dispersed in ultra pure water and then a few droplets of the slurry deposited on holey-carbon foils were mounted on conventional copper micro grids. This latter morphological characterization was realized only on CHA nanocrystals and $\mathrm{CHA}$ nanostructured microclusters contained in the Zn-CHA experimental toothpaste. 


\section{RAY DIFFRACTION ANALYSIS (XRD)}

$\mathrm{X}$-ray diffraction powder patterns were collected using an Analytical X'Pert Pro (Eindhoven, The Netherland) equipped with X'Celerator detector powder diffractometer using $\mathrm{Cu} \mathrm{K \alpha}$ radiation generated at $40 \mathrm{kV}$ and $40 \mathrm{~mA}$. The instrument was configured with a $1^{\circ}$ divergence and $0.2 \mathrm{~mm}$ receiving slits (X'PERT Guide, 2004).

The samples were prepared using the front-loading of standard aluminum sample holders, which are $1 \mathrm{~mm}$ deep, $20 \mathrm{~mm}$ high and $15 \mathrm{~mm}$ wide. The crystallinity degree was evaluated according to the following formula: crystallinity $=(\mathrm{X} / \mathrm{Y}) 100$ where $\mathrm{X}=$ net area of diffracted peaks, and $\mathrm{Y}=$ net area of diffracted peaks + background area.

\section{INFRARED MICROSCOPY SPECTRAL ANALYSIS (FT-IR)}

FT-IR spectra were recorded on a Thermo Nicolet 380 FT-IR spectrometer (Illinois, USA) equipped with a commercial ATR accessory. The infrared spectra were registered from 4000 to $400 \mathrm{~cm}^{-1}$ at $2 \mathrm{~cm}^{-1}$ resolution using a Bruker IFS 66v/S spectrometer using $\mathrm{KBr}$ pellets. Spectra were collected by averaging 32 scans at $4 \mathrm{~cm}^{-1}$ resolution.

\section{RESULTS}

\section{CARBONATE-HYDROXYAPATITE (CHA) NANOCRYSTALS AND CHA MICROCLUSTERS CHARACTERIZATION}

Figure 1 shows scanning and transmission electron micrographs of synthetic biomimetic CHA nanocrystals, with characteristic plate-like features and acicular morphology, and the aggregated CHA nanostructured microclusters.

The XRD of synthetic biomimetic CHA nanocrystals, showing the broadened diffraction maxima of an apatite single phase, is reported in Figure 2A. This pattern is compared with the XRD collected from the synthetic biomimetic CHA nanostructured microclusters used for preparing the $\mathrm{Zn}$-CHA toothpaste (Figure 2B) and the XRD of human tooth enamel (Figure 2C). There are evident similarities between Figures 2 A,B, whereas

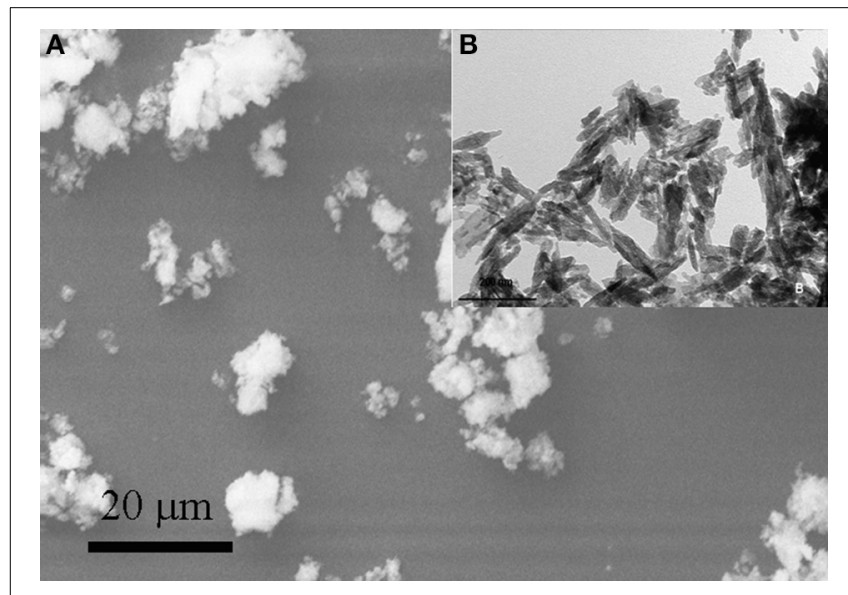

FIGURE 1 | Scanning electron micrograph of synthetic biomimetic CHA nanocrystals forming microclasters $(A)$, and the inset showing the transmission electron micrograph of $\mathrm{CHA}$ nanocrystals with characteristic acicular morphology (B).
Figure 2C shows the highest degree of crystallinity, proper of the natural enamel. The FT-IR spectra of synthesized CHA nanostructured microclusters and human enamel apatite are reported in Figures 3A,B, respectively. In both these spectra, it is possible to find characteristic bands at $1032-1104 \mathrm{~cm}^{-1}$, due to the phosphate group. The absorption band at $1472 \mathrm{~cm}^{-1}$ is related to the carbonate group substitution of the phosphate one, while the shoulder at $1550 \mathrm{~cm}^{-1}$ can be attributed to the carbonate group substituting the hydroxyl group in the apatite structure.

\section{ENAMEL SURFACE ANALYSIS}

A total of 12 teeth (one for each subject) were evaluated: five teeth extracted from subjects of the experimental group after the protocolled use of the Zn-CHA toothpaste, five teeth from subjects of the active control group after the protocolled use of $\mathrm{KNO}_{3} / \mathrm{NaF}$ toothpaste, two teeth from subjects that did not participate to the protocol (that brushed their teeth with a non-specified fluoride toothpaste, negative control). No complications after the extractions were observed in all the subjects enrolled in the present morphological in vivo investigation.

Figure 4A shows a scanning electron micrograph of the enamel surface of a tooth treated with $\mathrm{Zn}$-CHA toothpaste. In this micrograph, two different enamel superficial morphologies are evident: homogeneous smooth surface areas (black circle),

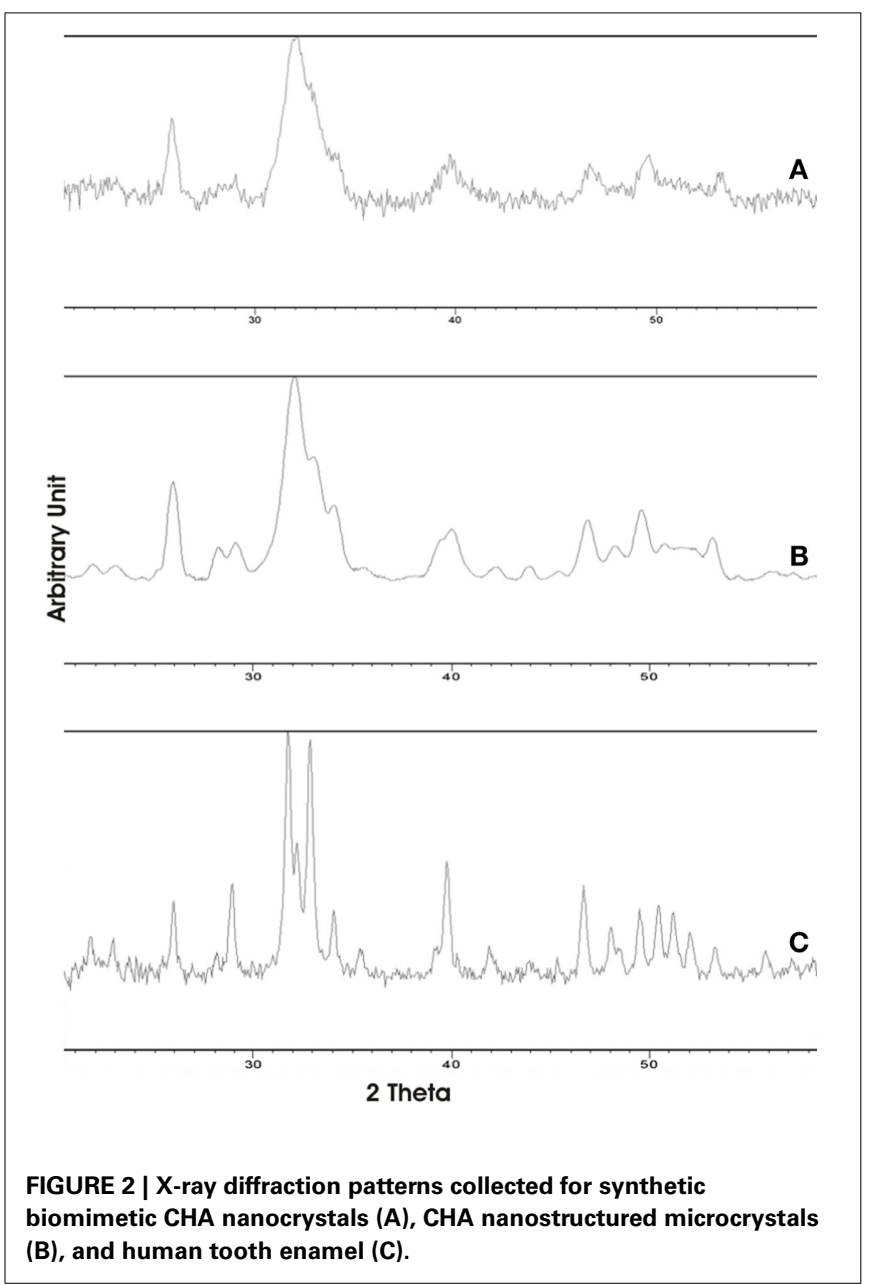


characterized by scraped surfaces, and areas of thin depositions (white circle). Figures 4B,C report the elementary analysis results obtained by EDAX probe pointed on surface areas labeled by black and white circles, respectively. No evidence of silica and fluoride can be observed, only the presence of calcium (C) and phosphorus $(\mathrm{P})$. The $\mathrm{Ca} / \mathrm{P}$ molar ratio results, calculated from Figures 4B,C, are 1.67 and 1.63 , respectively.

Figure 5A shows the scanning electron micrograph of the enamel surface of a tooth treated using $\mathrm{KNO}_{3} / \mathrm{NaF}$ toothpaste (positive control), in which two different areas can be discerned. In the first central area (white circle), there is a deposition of a phase mainly composed by silica and sodium, as shown in the elementary analysis results obtained by EDAX probe (Figure 5B). The presence of fluoride has not been detected. The elementary analysis results obtained by EDAX probe of the other area (black circle) (Figure 5C) reveal a $\mathrm{Ca} / \mathrm{P}$ molar ratio of 1.9 , which is the characteristic value of the native enamel hydroxyapatite (Wirsing et al., 1974; Hoyer et al., 1984).

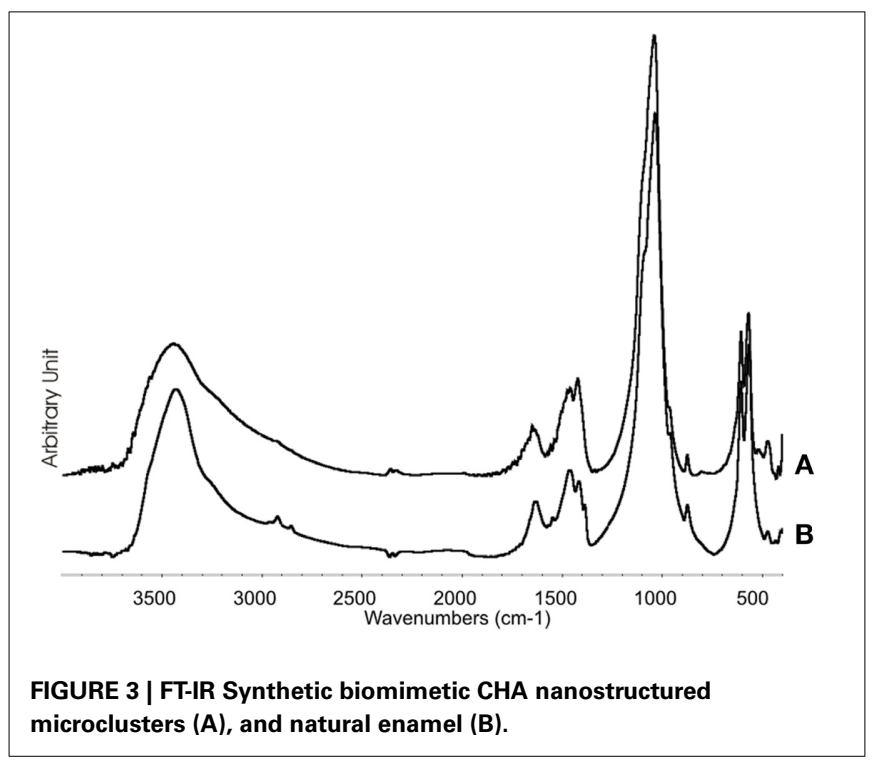

The enamel surface of a tooth representing the negative control, brushed with a non-specified fluoride toothpaste shows a zone of enamel loss (Figure 6).

Figure 7A shows the broadened X-Ray diffraction maxima of the synthetic biomimetic CHA microclusters of the $\mathrm{Zn}-\mathrm{CHA}$ experimental toothpaste. This XRD pattern can be compared with the ones obtained from enamel slabs of teeth treated using $\mathrm{Zn}$-CHA toothpaste, $\mathrm{KNO}_{3} / \mathrm{NaF}$ toothpaste and fluoride toothpaste, reported in Figures 7B-D, respectively. The XRD pattern obtained from enamel surfaces treated using $\mathrm{Zn}$-CHA toothpaste (Figure 7B) is similar to the one obtained from $\mathrm{Zn}$-CHA nanostructured microclusters (Figure 7A), thus revealing its deposition on the enamel surface.

On the other hand, the XRD pattern recorded on the enamel surfaces obtained from teeth treated using $\mathrm{KNO}_{3} / \mathrm{NaF}$ toothpaste (Figure 7C) appear slightly less sharpened than those obtained from the surface of enamel treated using the fluoride toothpaste (Figure 7D). Figure 8A shows the FT-IR absorption pattern of the enamel surface of teeth treated with $\mathrm{Zn}$-CHA toothpaste, which is similar to the one of the enamel HA (Roveri et al., 2009b).

The analysis of the FT-IR absorption phosphate bands at about 1030 - $1090 \mathrm{~cm}^{-1}$ shows the different degree of crystallinity relative to the different enamel samples analyzed. In fact, the adsorption bands reported in Figure 8A demonstrate a low degree of resolution of the phosphate peak in the enamel treated using the CHA-based toothpaste. On the other hand, the FT-IR absorption pattern obtained in the enamel treated using the $\mathrm{KNO}_{3} / \mathrm{NaF}$ toothpaste (Figure 8B) reveals the presence of a peak at $3573 \mathrm{~cm}^{-1}$, which is characteristic of the substitution of hydroxyl groups with fluoride ions, into the hydroxyapatite structure (Wang et al., 2009). This peak is also visible in the FT-IR absorption pattern obtained in the spectrum of enamel in vivo treated with the fluoride toothpaste (Figure 8C).

\section{DISCUSSION}

Hydroxyapatite, in enamel and in bone, is responsible for the mechanical behavior of the calcified tissues. Unlike bone, when the enamel hydroxyapatite is dissolved or abraded, it cannot spontaneously remineralize, because enamel contains no cells

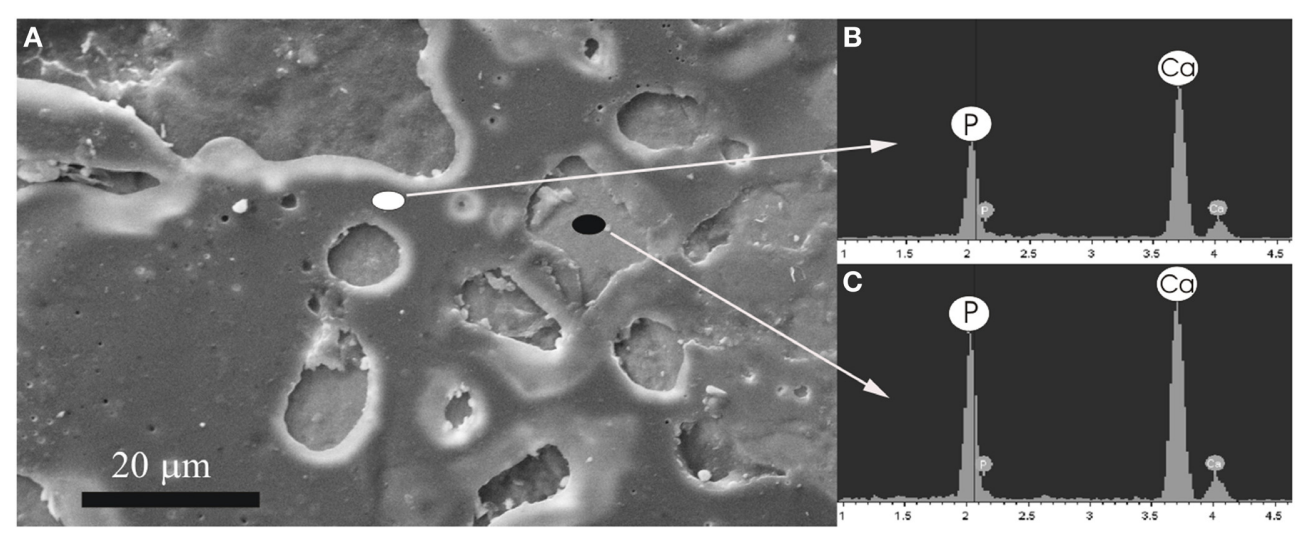

FIGURE 4 | Scanning electron micrograph of the enamel surface of a tooth in vivo treated using the Zn-CHA toothpaste (A), EDAX results using a probe pointed in the area labeled by the white circle (B), EDAX results using a probe pointed in the area labeled by the black circle (C). 


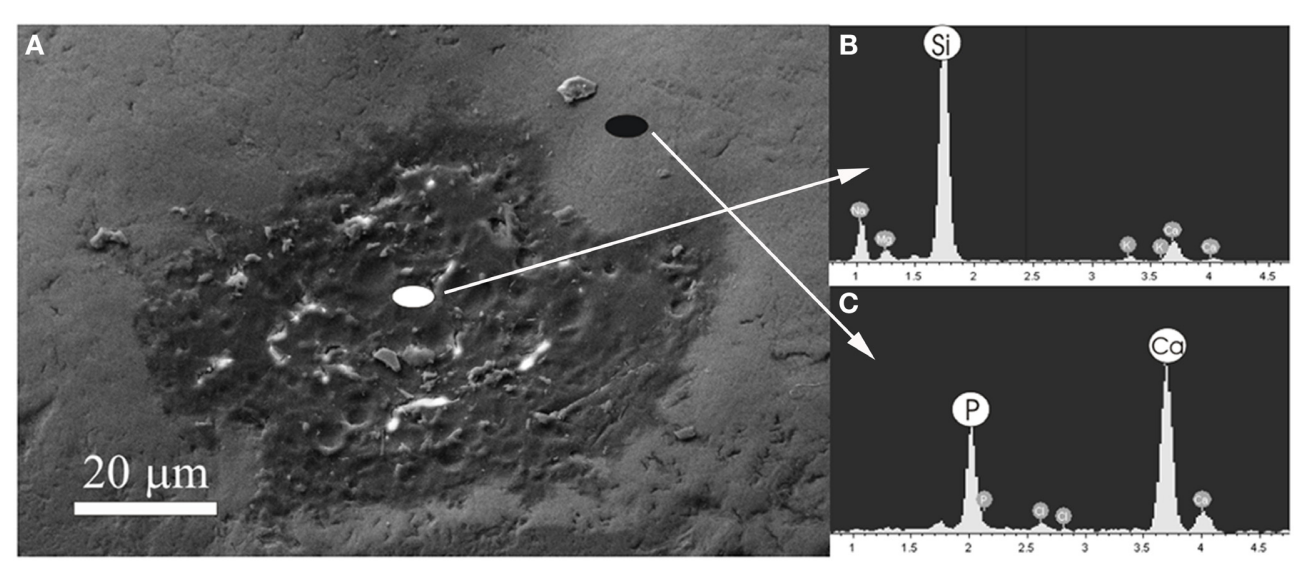

FIGURE 5 | Scanning electron micrograph of the enamel surface of a tooth in vivo treated using the $\mathrm{KNO}_{3} / \mathrm{NaF}$ toothpaste (A), EDAX with probe pointed in the area labeled by the white circle (B), EDAX with probe pointed in the area labeled by the black circle (C).

(Roveri et al., 2009a). Biomimetic carbonate hydroxyapatite (CHA) nanocrystals have been synthesized with a stoichiometric $\mathrm{Ca} / \mathrm{P}$ molar ratio of about $1.7 \pm 0.1$, containing $4 \pm 1 \mathrm{wt} \%$ of carbonate ions, prevalently replacing phosphate groups, while $1 \%$ of $\mathrm{Ca}^{2+}$ ions are substituted by $\mathrm{Zn}^{2+}$. The nanostructured $\mathrm{Zn}$-CHA microcrystals, prepared in laboratory according to the patented methodology, represent the active component of the experimental $\mathrm{Zn}$-CHA toothpaste. The micrometric dimension of the crystal clusters allows avoiding any suspicion about the in vivo utilization of nano-dimensioned particles. Nevertheless, the nanostructured surface of the microclusters is responsible for the high surface area that is crucial for their chemical reactivity.

Synthesized biomimetic CHA nanocrystals and human enamel apatite not only contain a similar carbonate amount, but also have been shown to promote carbonate substitution to the phosphate and/or hydroxyl group, which is very similar to the synthetic and biological CHA nanocrystals. The synthetic experimental CHA nanocrystals have a plate-like morphology and a structure very close to that of the enamel, dentine and bone hydroxyapatite, and exhibiting very similar FT-IR spectra, even if enamel spectrum shows the highest degree of crystallinity according to previous findings (Teaford, 1988).

In summary, in the present work, the surface of teeth treated in vivo for 8 weeks with: (i) Zn-CHA toothpaste (experimental group); (ii) $\mathrm{KNO}_{3} / \mathrm{NaF}$ toothpaste (active control group); (iii) fluoride toothpaste (negative control group) have been compared, by means of SEM-EDAX investigation, XRD, and FT-IR.

SEM-EDAX, XRD, and FT-IR observations showed that, after the in vivo treatment using the $\mathrm{Zn}$ - $\mathrm{CHA}$ toothpaste, an appreciable formation of a biomimetic CHA coating was present on the enamel surface. On the other hand, not any mineral deposition, in spite of silica, has been observed after the in vivo treatment using both a $\mathrm{KNO}_{3} / \mathrm{NaF}$ toothpaste and non-specified fluoride toothpaste. The in vivo treatment with these two latter toothpastes changes the structure of enamel natural hydroxyapatite crystals, increasing their degree of crystallinity (XRD investigation), by means of the partial substitution of hydroxyl groups with fluoride ions (FT-IR investigation). Indeed, the phosphate

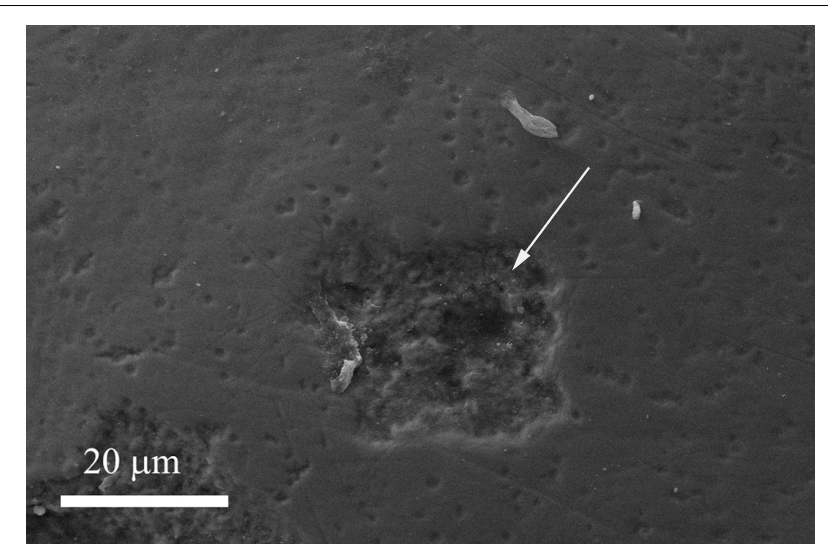

FIGURE 6 | Scanning electron micrograph of the enamel surface treated using a non-specified fluoride toothpaste, showing a zone of enamel loss (arrow).

band in the enamel surface after treatment with $\mathrm{KNO}_{3} / \mathrm{NaF}$ or a non-specified fluoride toothpaste appears highly solved respect to the one of the phosphate group reported in Figure $\mathbf{8 A}$, thus demonstrating the high degree of crystallinity of these enamel surfaces.

On the other hand, the remineralizing/repairing effect of the enamel surface treated using synthetic nanostructured CHA microcrystals is consistent with a mineral biomimetic apatitic deposition, which does not alter the chemical-physic properties of the enamel. The biomimetic CHA coating can appear of different thickness, probably due to the underside different enamel surface morphology, which can change in function of the degree of enamel damage. However, the EDAX analysis reveals that the $\mathrm{Ca} / \mathrm{P}$ molar ratio of CHA crystals (about 1.7) is homogeneously constant on the enamel surface. This finding assures a uniform enamel protection against the enamel wear and loss phenomena, thus preventing dentine exposure.

Results of the first clinical randomized trial by Orsini et al. (2010) have already demonstrated the efficacy of $\mathrm{Zn}-\mathrm{CHA}$ toothpastes in reducing $\mathrm{DH}$. Moreover, a further very recent 


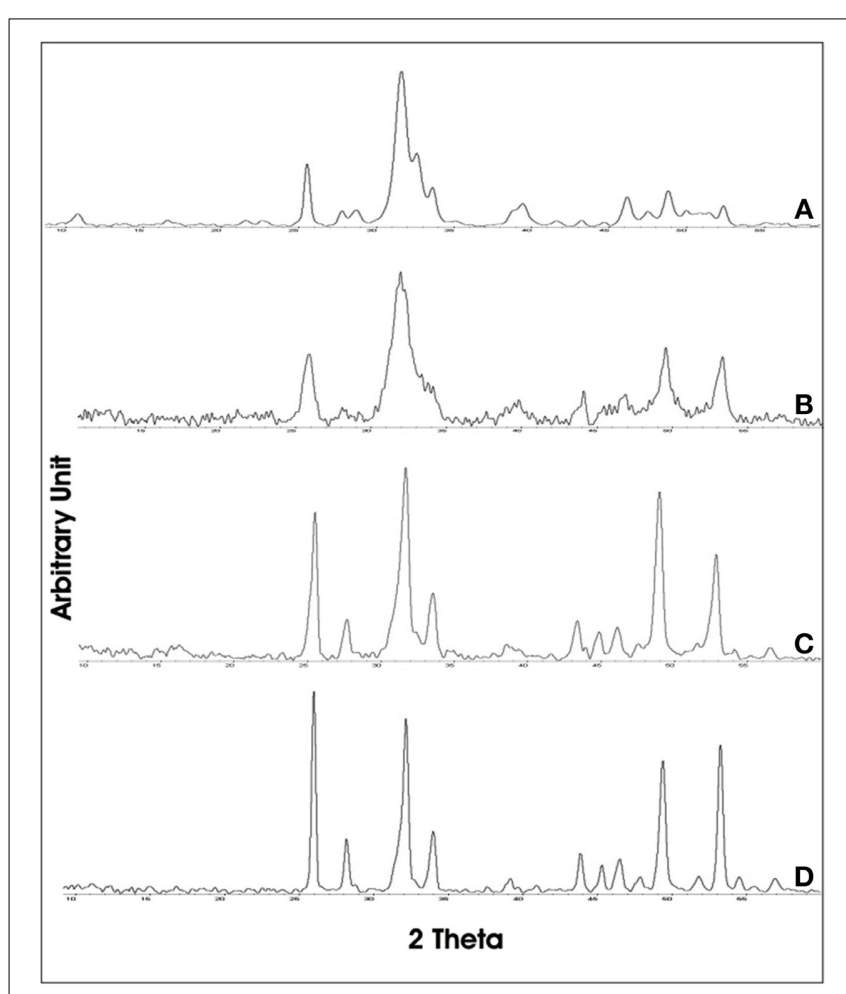

FIGURE 7 | DRX pattern of the enamel after the in vivo treatment using the following. (A) synthetic biomimetic $\mathrm{CHA}$ nanostructured microclusters, (B) $\mathrm{Zn}-\mathrm{CHA}$ toothpaste, (C) $\mathrm{KNO} / \mathrm{NaF}$ toothpaste, (D) Fluoride toothpaste.

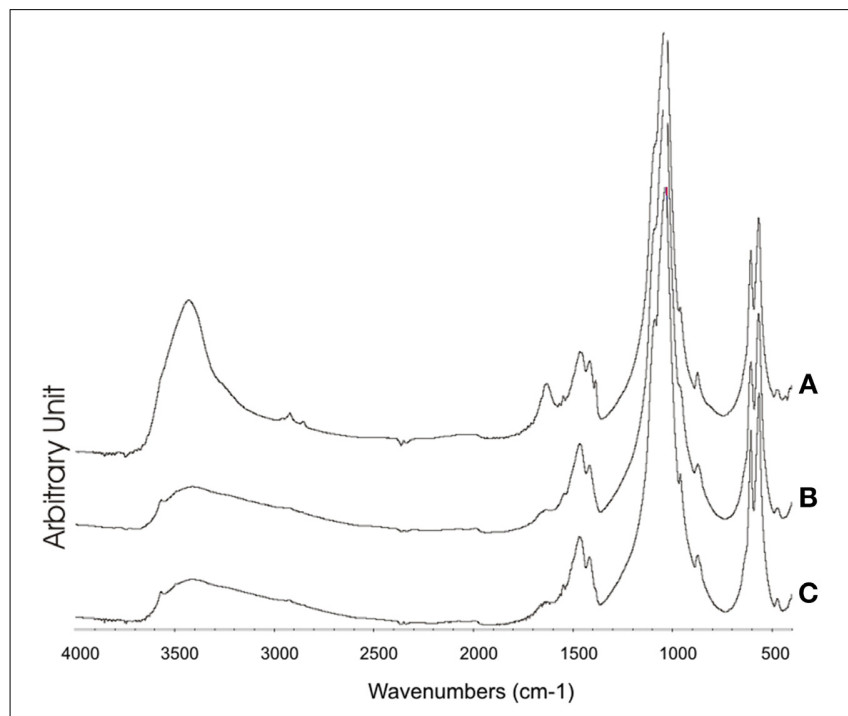

FIGURE 8 | FT-IR of the enamel surface after treatment using. (A) $\mathrm{Zn}-\mathrm{CHA}$ containing toothpaste, (B) $\mathrm{KNO}_{3} / \mathrm{NaF}$ toothpaste, (C) Fluoride toothpaste.

randomized trial by the same authors showed that this effect could be exerted after only 3 days of treatment (Orsini et al., 2013).

The results of this in vivo morphological and chemical-physic study might in part explain the beneficial effect of $\mathrm{Zn}-\mathrm{CHA}$ toothpastes in reducing $\mathrm{DH}$, since the deposition of a synthetic nanostructured CHA microcrystals-rich coating could lead to a remineralizing/repairing effect of the enamel surface, in the teeth treated using $\mathrm{Zn}$-CHA toothpaste. Therefore, the principal finding of this study is that: (1) the remineralizing mechanism of the nanostructured CHA microcrystals, largely documented by previous in vitro reports (Roveri et al., 2008, 2009a) can be also confirmed in vivo. Moreover, it can be suggested that this synthetic CHA deposition mainly occurs on the enamel areas characterized by enamel loss and/or damage (probably due to erosion effects), thus being considered as a real enamel repair (see Figure 4).

In contrast, (2) the use of a toothpaste containing $\mathrm{KNO}_{3} / \mathrm{NaF}$ may form only a deposition, consisting of silica, as an abrasive phase on the enamel surface, which, however, does not remineralize the damaged enamel area, but it is generally deposed in correspondence of natural concavities proper of the natural tooth morphology (see Figure 5). Furthermore, no deposition on the enamel surfaces has been observed after treatment using the fluoride and the $\mathrm{KNO}_{3} / \mathrm{NaF}$ toothpastes (see Figure 6), which may lead only to a partial substitution of the hydroxyl groups with fluoride ions in the native enamel hydroxyapatite.

The CHA formed coating is generally insoluble in physiological mouth $\mathrm{pH}$, but it may undergo to solubilization when, for instance, a bacterial biofilm covers the teeth and its products decrease the $\mathrm{pH}$ value. During the CHA coating solubilization, $\mathrm{Ca}$ ions, phosphates, and $\mathrm{Zn}$ ions are released, allowing the $\mathrm{Zn}$ to exploit a strong antibacterial effect, which interferes with the plaque formation, thus preventing further solubilization processes of the newly deposed $\mathrm{Zn}$-CHA coating. Therefore, it may be suggested that the coating formed by $\mathrm{Zn}$ - CHA toothpastes may exploit not only a remineralizing effect of the dental surface, but also a beneficial effect toward bacterial plaque attacks.

The main limitation of this work is that the in vivo remineralizing effect exploited by the Zn-CHA toothpaste was morphologically demonstrated only on the enamel surfaces, since the analyzed extracted teeth did not present areas of dentine exposition. Therefore, further studies will be carried out in vivo to analyse whether a stable biomimetic CHA deposition (Roveri et al., 2009a) and a repairing mechanism can be demonstrated also in dentinal surfaces.

In conclusion, the present study shows that only the use of a toothpaste containing $\mathrm{Zn}$-substituted CHA nanocrystals can produce a biomimetic coating on the enamel surface, thus mimicking the composition, structure, morphology and surface reactivity of the biological enamel hydroxyapatite.

\section{ACKNOWLEDGMENTS}

We thank the Universities of Bologna and Polytechnic of Marche (funds for selected research topics), the Chemical Center s.r.l. (for technical support and instrumental facilities), the Inter University Consortium for Research on Chemistry of Metals in Biological Systems (C.I.R.C.M.S.B) and RBAP114AMK, RINAME Project, "Rete integrata per la nano medicina" (funds for selected research topics) Coswell S.P.A., Funo, Bologna, Italy is acknowledged for providing toothpastes. 


\section{REFERENCES}

X'PERT Guide. (2004). X'Pert High Score Plus 2.0a. Almelo: Panalytical B.V.

Absi, E. G., Addy, M., and Adams, D. (1987). Dentine hypersensitivity. A study of the patency of dentinal tubules in sensitive and non-sensitive cervical dentine. J. Clin. Periodontol. 14, 280-284. doi: 10.1111/j.1600-051X.1987.tb01533.x

Addy, M. (1990). Etiology and clinical implications of dentine hypersensitivity. Dent. Clin. North Am. 34, 503-514.

Addy, M., and West, N. X. (2013). The role of toothpaste in the aetiology and treatment of dentine hypersensitivity. Monogr. Oral Sci. 23, 75-87. doi: $10.1159 / 000350477$

Addy, M., West, N. X., Barlow, A., and Smith, S. (2007). Dentine hypersensitivity: is there both stimulus and placebo responses in clinical trials? Int. J. Dent. Hyg. 5, 53-59. doi: 10.1111/j.1601-5037.2007.00228.x

Bellamy, P. G., Khera, N., Day, T. N., Barker, M. L., and Mussett, A. J. (2009). A randomized clinical trial to compare plaque inhibition of a sodium fluoride/potassium nitrate dentifrice versus a stabilized stannous fluoride/sodium hexametaphosphate dentifrice. J. Contemp. Dent. Pract. 10, 1-9.

Brannström, M. (1963). "A hydrodynamic mechanism in the transmission of pain producing stimuli through dentine," in Sensory Mechanisms in Dentine, ed D. J. Anderson (Oxford: Pergamon Press), 73-79.

Dababneh, R. H., Khouri, A. T., and Addy, M. (1999). Dentine hypersensitivity an enigma? a review of terminology, epidemiology, mechanisms, aetiology and management. Br. Dent. J. 187, 606-611.

Drisko, C. (2007). Oral hygiene and periodontal considerations in preventing and managing dentine hypersensitivity. Int. Dent. J. 57, 399-410. doi: 10.1111/j.1875-595X.2007.tb00167.x

Gazzaniga, G., Roveri, N., Rimondini, L., Palazzo, B., Iafisco, M., and Gualandi, P. (2006). Biologically Active Nanoparticles of a Carbonate-Substituted Hydroxyapatite Process for Their Preparation and Compositions Incorporating the Same. Patent:WO2007137606. Available online at: http://www.google.com/ patents/WO2007137606A1?cl=en\&hl=it

Groeneveld, A., Van, E. A. A., and Backer, D. O. (1990). Fluoride in caries prevention: is the effect pre- or post-eruptive? J. Dent. Res. 69, 751-755.

Hefferren, J. J. (1976). Radioactive dentine abrasion. J. Dent. Res. 55, 563-573.

Holland, G. R., Narhi, M. N., Addy, M., Gangarosa, L., and Orchardson, R. (1997). Guidelines for the design and conduct of clinical trials on dentine hypersensitivity. J. Clin. Periodontol. 24, 808-813.

Hooper, S., West, N. X., Pickles, M. J., Joiner, A., Newcombe, R. G., and Addy, M. (2003). Investigation of erosion and abrasion on enamel and dentine: a model in situ using toothpastes of different abrasivity. J. Clin. Periodontol. 30, 802-808. doi: 10.1034/j.1600-051X.2003.00367.x

Hoyer, I., Gaengler, P., and Bimberg, R. (1984). In vivo remineralization of human enamel and dental calculus formation. J. Dent. Res. 63, 1136-1139.

Kuroiwa, M., Kodaka, T., Kuroiwa, M., and Abe, M. (1994). Dentin hypersensitivity. Occlusion of dentinal tubules by brushing with and without an abrasive dentifrice. J. Periodontol. 65, 291-296.

Lee, S. Y., Kwon, H. K., and Kim, B. I. (2008). Effect of dentinal tubule occlusion by dentifrice containing nano-carbonate apatite. J. Oral Rehabil. 35, 847-853. doi: 10.1111/j.1365-2842.2008.01876.x

Markowitz, K., Bilotto, G., and Kim, S. (1991). Decreasing intradental nerve activity in the cat with potassium and divalent cations. Arch. Oral Biol. 36, 1-7.

Nagata, T., Ishida, H., Shinohara, H., Nishikawa, S., Kasahara, S., Wakano, Y., et al. (1994). Clinical evaluation of a potassium nitrate dentifrice for the treatment of dentinal hypersensitivity. J. Clin. Periodontol. 21, 217-221.

Orchardson, R., and Gillam, D. G. (2000). The efficacy of potassium salts as agents for treating dentin hypersensitivity. J. Orofac. Pain 14, 9-19.

Orsini, G., Procaccini, M., Manzoli, L., Giuliodori, F., Lorenzini, A., and Putignano, A. (2010). A double-blind randomized-controlled trial comparing the desensitizing efficacy of a new dentifrice containing carbonate/hydroxyapatite nanocrystals and a sodium fluoride/potassium nitrate dentifrice. J. Clin. Periodontol. 37, 510-517. doi: 10.1111/j.1600-051X.2010. 01558.x

Orsini, G., Procaccini, M., Manzoli, L., Sparabombe, S., Tiriduzzi, P., Bambini, F., et al. (2013). A 3-day randomized clinical trial to investigate the desensitizing properties of three dentifrices. J. Periodontol. 84, e65-e73. doi: 10.1902/jop.2013.120697

Peacock, J. M., and Orchardson, R. (1995). Effects of potassium ions on action potential conduction in A- and C-fibers of rat spinal nerves. J. Dent. Res. 74, 634-641.
Poulsen, S., Errboe, M., Lescay Mevil, Y., and Glenny, A. M. (2006). Potassium containing toothpastes for dentine hypersensitivity. Cochrane Database Syst. Rev. 3, CD001476. doi: 10.1002/14651858

Rimondini, L., Palazzo, B., Iafisco, M., Canegallo, L., Demarosi, F., Merlo, M., et al. (2007). The remineralizing effect of carbonate-hydroxyapatite nanocrystals on dentine. Mater. Sci. Forum 539-543, 602-605. doi: 10.4028/www.scientific.net/MSF.539-543.602

Roveri, N., Battistella, E., Foltran, I., Foresti, E., Iafasco, M., Lelli, M. et al. (2008). Synthetic biomimetic carbonate-hydroxyapatite nanocrystals for enamel remineralization. Adv. Mater. Res. 47-50, 821-824. doi: 10.4028/www.scientific.net/AMR.47-50.821

Roveri, N., Battistella, E., Bianchi, C. L., Foltran, I., Foresti, E., Iafasco, M., et al. (2009a). Surface enamel remineralization: biomimetic apatite nanocrystals and fluoride ions different effects. J. Nanomater. 2009, 1-9. doi: $10.1155 / 2009 / 746383$

Roveri, N., Foresti, E., Lelli, M., and Lesci, I. G. (2009b). Recent advancements in preventing teeth health hazard: the daily use of hydroxyapatite instead of fluoride. Rec. Patents Biomed. Eng. 2, 197-215. doi: 10.2174/18747647109020 30197

Scheutzel, P. (1996). Etiology of dental erosion intrinsic factors. Eur. J. Oral Sci. 104, $178-190$.

Silverman, G., Berman, E., Hanna, C. B., Salvato, A., Fratarcangelo, P. Bartizek, R. D., et al. (1996). Assessing the efficacy of three dentifrices in the treatment of dentinal hypersensitivity. J. Am. Dent. Assoc. 127, 191-201.

Singal, P., Gupta, R., and Pandit, N. (2005). 2\% Sodium fluoride-iontophoresis compared to a commercially available desensitizing agent. J. Periodontol. 76, 351-357. doi: 10.1902/jop.2005.76.3.351

Smith, B. G. N., and Knight, J. K. (1984). A comparison of patterns of toothwear with the etiological factors. Br. Dent. J. 157, 16-19.

Tarbet, W. J., Silverman, G., and Stolman, J. M. (1979). An evaluation of two methods for the quantitation of dentinal hypersen-sitivity. J. Am. Dent. Assoc. 98, 914-918.

Teaford, M. F. (1988). A review of a dental microwear and diet in modern mammals. Scan. Microsc. 2, 1149-1166.

Van't Spijker, A., Rodriguez, J. M., Kreulen, C. M., Bronkhorst, E. M., Bartlett, D. W., and Creugers, N. H. (2009). Prevalence of tooth wear in adults. Int. J. Prosthodont. 22, 35-42.

Wang, J., Chao, Y., Wan, Q., Yan, K., and Meng, Y. (2009). Fluoridated hydroxyapatite/titanium dioxide nanocomposite coating fabricated by a modified electrochemical deposition. J. Mater. Science Mater. Med. 20, 1047-1055. doi: 10.1007/s10856-008-3673-1

Wirsing, A., Judd, G., and Ansell, G. S. (1974). Electron probe microanalysis of human enamel microstructure. J. Dent. Res. 53, 491-494.

Zero, D. T., and Lussi, A. (2000). "Etiology of enamel erosion: intrinsic and extrinsic factors," in Tooth Wear and Sensitivity, eds M. Addy, G. Embery, W. M. Edgar, and R. Orchardson (London: Martin Dunitz), 121-140.

Conflict of Interest Statement: The authors declare that the research was conducted in the absence of any commercial or financial relationships that could be construed as a potential conflict of interest.

Received: 15 July 2014; paper pending published: 27 July 2014; accepted: 13 August 2014; published online: 05 September 2014.

Citation: Lelli M, Putignano A, Marchetti M, Foltran I, Mangani F, Procaccini M, Roveri $N$ and Orsini $G$ (2014) Remineralization and repair of enamel surface by biomimetic Zn-carbonate hydroxyapatite containing toothpaste: a comparative in vivo study. Front. Physiol. 5:333. doi: 10.3389/fphys.2014.00333

This article was submitted to Craniofacial Biology, a section of the journal Frontiers in Physiology.

Copyright (C) 2014 Lelli, Putignano, Marchetti, Foltran, Mangani, Procaccini, Roveri and Orsini. This is an open-access article distributed under the terms of the Creative Commons Attribution License (CCBY). The use, distribution or reproduction in other forums is permitted, provided the original author(s) or licensor are credited and that the original publication in this journal is cited, in accordance with accepted academic practice. No use, distribution or reproduction is permitted which does not comply with these terms. 